\title{
Telehealth Utilization Is Associated with Lower Risk of Discontinuation of Buprenorphine: a Retrospective Cohort Study of US Veterans
}

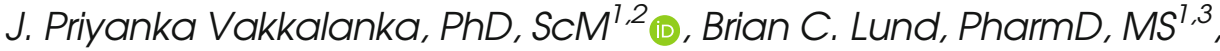 \\ Marcia M. Ward, PhD ${ }^{4}$, Stephan Arndt, $P h D^{5,6}$, R. William Field, $P h D^{7}$, \\ Mary Charlton, $P h D^{7}$, and Ryan M. Carnahan, PharmD, $M S^{l}$
}

\begin{abstract}
'Department of Epidemiology, University of lowa College of Public Health, lowa City, IA, USA; ${ }^{2}$ Department of Emergency Medicine, University of lowa Carver College of Medicine, lowa City, IA, USA; ${ }^{3}$ Center for Comprehensive Access and Delivery Research and Evaluation, lowa City Veterans Affairs Health Care System, lowa City, IA, USA; ${ }^{4}$ Department of Health Management and Policy, University of lowa College of Public Health, lowa City, IA, USA; ${ }^{5}$ Department of Biostatistics, University of lowa College of Public Health, lowa City, IA, USA; ${ }^{6}$ Department of Psychiatry, University of lowa Carver College of Medicine, lowa City, IA, USA; ${ }^{7}$ Department of Occupational and Environmental Health, University of lowa College of Public Health, lowa City, IA, USA.
\end{abstract}

OBJECTIVE: Treatment for opioid use disorder (OUD) may include a combination of pharmacotherapies (such as buprenorphine) with counseling services if clinically indicated. Medication management or engagement with in-person counseling services may be hindered by logistical and financial barriers. Telehealth may provide an alternative mechanism for continued engagement. This study aimed to evaluate the association between telehealth encounters and time to discontinuation of buprenorphine treatment when compared to traditional inperson visits and to evaluate potential effect modification by rural-urban designation and in-person and telehealth combination treatment.

METHODS: A retrospective cohort study of Veterans diagnosed with OUD and treated with buprenorphine across all facilities within the Veterans Health Administration (VHA) between 2008 and 2017. Exposures were telehealth and in-person encounters for substance use disorder (SUD) and mental health, treated as timevarying covariates. The primary outcome was treatment discontinuation, evaluated as 14 days of absence of medication from initiation through 1 year.

RESULTS: Compared to in-person encounters, treatment discontinuation was lower for telehealth for SUD (aHR: 0.69; 95\%CI: 0.60, 0.78) and mental health (aHR: 0.69; 95\%CI: 0.62, 0.76). There was no evidence of effect modification by rural-urban designation. Risk of treatment discontinuation appeared to be lower among those with telehealth only compared to in-person only for both SUD (aHR: 0.48, 95\%CI: 0.37, 0.62) and for mental health (aHR: 0.46; 95\%CI: 0.33, 0.65).

CONCLUSIONS: As telehealth demonstrated improved treatment retention compared to in-person visits, it may be a suitable option for engagement for patients in OUD management. Efforts to expand services may improve

Previous Presentation: None

Received February 11, 2021

Accepted June 4, 2021

Published online June 22, 2021 treatment retention and health outcomes for VHA and other health care systems.

KEY WORDS: Veterans health; treatment adherence and compliance; buprenorphine; telehealth; rural health.

$\begin{array}{ll}\text { Abbreviations } \\ \text { OUD } & \text { Opioid use disorder } \\ \text { aHR } & \text { Adjusted hazard ratio } \\ \text { VHA } & \text { Veterans Health Administration } \\ \text { ICD-9/ } & \text { International Classification of Diseases - Clinical } \\ 10 & \text { Modification Versions } 9 \text { and } 10 \text { (ICD-9-CM, ICD-10) } \\ \text { PTSD } & \text { Post-traumatic stress disorder }\end{array}$

J Gen Intern Med 37(7):1610-8

DOI: $10.1007 / \mathrm{s} 11606-021-06969-1$

(C) Society of General Internal Medicine 2021

\section{INTRODUCTION}

Opioid use disorder (OUD) has had a significant impact in the United States (US) with nearly 450,000 people dying from an overdose involving opioids between 1999 and 2018. ${ }^{1}$ Veterans in the US represent a unique cohort that is at risk for OUD due to their greater rates of concurrent substance use, psychiatric comorbidities, trauma, and homelessness when compared to the general population. ${ }^{2-9}$ Among US Veterans, the estimates of diagnosed OUD increased to over 69,132 in 2017 from 25,031 in $2003 . .^{10,11}$

Treatment for OUD is available through pharmacotherapies (such as buprenorphine, buprenorphine/naloxone, methadone, or naltrexone), and behavioral interventions including counseling. ${ }^{12}$ The added component for optional or ancillary psychological services for those who need it the most, however, may be cumbersome depending on the frequency and duration of treatment, availability of counselors and providers, and distance to the provider. ${ }^{13-16}$ This could subsequently influence retention in treatment or length of stay in treatment, which is the most consistent predictor of post-treatment 
outcomes. ${ }^{17,18}$ Continued engagement in counseling services, however, may especially be challenging for rural communities or Veterans living in rural areas. ${ }^{11,19}$ Rural areas struggle with long waiting lists for MOUD, and the combination of high rates of opioid prescriptions with fewer treatment options is a continued challenge. ${ }^{20}$

Telehealth, the use of electronic communication and information technologies to provide or support clinical care, has increased as a service to overcome logistical, financial, and stigma-related challenges in mental health care. ${ }^{21-23} \mathrm{~A}$ review of telehealth services within the Veterans Health Administration (VHA) noted three key findings: (1) telehealth utilization increased over 300\% between 2009 and 2015; (2) utilization proliferated most significantly among rural Veterans; and (3) approximately $50 \%$ of telehealth encounters were for mental health care. ${ }^{24}$ Telehealth for OUD management has gained momentum in recent years due to shortages of certified providers in rural areas and logistical barriers of in-person visits. If comparable to in-person counseling in improving OUD outcomes through buprenorphine treatment retention, telehealth could be considered as an additional means to provide care. In this study, we aimed to investigate whether the incorporation of telehealth services for Veterans treated for OUD within the VHA was associated with buprenorphine treatment retention and to evaluate whether this association varied between rural and urban Veterans and those with combined inperson and telehealth visits.

\section{METHODS}

\section{Study Design, Setting, and Sample}

We conducted a retrospective cohort study of adult Veterans $(\geq 18$ yo) diagnosed with OUD and treated with buprenorphine or buprenorphine/naloxone within the VHA system between January 1, 2008, and December 31, 2017. Eligible Veterans included those who received an incident prescription for a sublingual, short-acting buprenorphine product and had a diagnosis code for OUD using the International Classification of Diseases - Clinical Modification Versions 9 and 10 within 6 months of the prescription. We excluded those receiving buprenorphine patches as these suggest pain management, those with a documentation of a metastatic tumor diagnosis (as this may influence subsequent medications, treatment retention, and mortality) within 2 years prior to buprenorphine initiation, and those without geographical information on rurality. This study was approved by the University of Iowa Institutional Review Board and the Iowa City Veterans Administration Research and Development Committee and is reported according to STROBE guidelines. ${ }^{25}$

\section{Measurement of Telehealth and Other Visits}

Within the cohort of Veterans identified for OUD management with buprenorphine, we identified those who had telehealth services that occurred during the course of treatment through the Decision Support System identifier. ${ }^{26}$ These stop codes are used by the VHA to evaluate clinical workload with a physical location for that service, help define outpatient production units, and estimate the costs of outpatient VHA work. Telehealth was defined using a strict definition that included stop codes indicating clinic-to-clinic (CCVT) or clinic-to-home (CHVT) video telehealth derived from previous work by Adams et al. and other resources (Appendix A). ${ }^{24,27}$ We identified stop codes specifically for substance use disorder (SUD) counseling and services as the primary exposure, and a broader, general mental health services as a secondary exposure that encompassed SUD and other mental health services. ${ }^{24}$ Both SUD and mental health exposure variables were coded as telehealth (whether alone or in combination with in-person encounters), in-person only, or a gap between encounters. As there is no specific rule or guideline for the frequency of therapy visits for OUD management, we determined that at least one encounter or contact every 4 weeks with the VHA would be the minimum needed to stay engaged. The exposure was evaluated as a time-varying covariate such that each telehealth encounter was the start date of a 28-day period in which the Veteran was considered exposed to telehealth, and the start date was reset with each new telehealth visit (Appendix B).

\section{Measurement of Covariates and Confounders}

To account for temporal trends in buprenorphine availability and access, we categorized the year the Veteran initiated buprenorphine within 2-year intervals. Sociodemographic factors assessed at baseline included age, race, ethnicity, gender, and potential homelessness (identified using previous methodologies). ${ }^{28,29}$ Geographical measures included driving distance and time to nearest primary or secondary care centers and urban/rural designation status determined by 2010 RuralUrban Commuting Area codes. ${ }^{30}$

Co-morbidities diagnosed up to 2 years prior to buprenorphine initiation were identified from ICD-9 and ICD-10 inpatient and outpatient diagnosis codes using the approach of Quan et al., and the Clinical Classifications Software (CCS) Level 2 coding schema for mental health diagnoses. ${ }^{31,32} \mathrm{We}$ captured past year psychiatric medications in addition to those for OUD. Prior healthcare utilization was measured as admissions or encounters for inpatient (any, mental health, suicide, or substance use), outpatient, urgent care, and emergency department utilization from VA stop codes up to 2 years before buprenorphine initiation. ${ }^{33}$

\section{Outcome Assessment}

The primary outcome in this study was time to treatment discontinuation (days). The incident buprenorphine date for each Veteran was determined from the first sublingual buprenorphine outpatient prescription filled between January 1, 2008, and December 31, 2017. An episode of treatment was 
generated by assessing the supply days from the day the buprenorphine outpatient prescription was filled through the number of supply days, and we accounted for potential oversupply if the next prescription was filled prior to the expected exhaustion of supply. To account for gaps in treatment due to hospitalization, incarceration, or logistical factors, we defined treatment discontinuation as a gap of greater than 14 days.

\section{Statistical Analysis}

We evaluated telehealth encounters for SUD or mental health until treatment discontinuation. Veterans were censored at a transition to another medication for OUD $(n=167)$, at the end of the study period (December 31, 2017), or end of maximum follow-up period of 1 year. Deaths occurring during and shortly after treatment discontinuation were handled in one of two ways. Those who died during the treatment episode were followed for the number of days in treatment until death, at which point they were censored $(n=95)$. Those who died within 14 days of depletion of the buprenorphine supply were classified as having discontinued treatment at the time of death $(n=44)$.

The association between each demographic and clinical covariate and treatment discontinuation was modeled through Cox proportional hazards regression. Variables identified as associated with the outcome (with a threshold of $p<0.20$ ) were considered in developing the final model. Final multivariable models were built through the inclusion of variables that were associated with the exposure and outcome to account for confounding of telehealth-related effect estimates and evaluation of Akaike information criterion values. All analyses were completed using SAS version 9.4 (Cary, NC).

Secondary Analyses. We evaluated potential multiplicative effect modification of treatment and outcome associations by rural-urban categorization. Second, Veterans were further classified as having been exposed to telehealth only, a combination of telehealth and in-person visits, or in-person visits only in the prior 28 days.

Sensitivity Analyses. We performed multiple sensitivity analyses of outcomes. First, we addressed the issue of new users within the VHA having incomplete co-morbidity data by excluding those at or below the $10^{\text {th }}$ percentile of outpatient visits in the 2-year baseline period. Second, we analyzed Veterans who stayed in treatment for $\geq 28$ days to account for those who discontinued treatment early and might not have had the opportunity to initiate telehealth. As certain facilities might have adopted telehealth for patient care earlier or more aggressively than other sites, there may have also been other programs or interventions within these facilities that might have explained the associations for telehealth. In order to account for this issue, we limited one analysis to the 10 sites that most frequently used telehealth.

For potential exposure misclassification, we changed the exposed window of 28 to 14 days. For outcome misclassification, we examined alternative durations of apparent lapses in treatment to define discontinuation as 7,28, and 90 days of nontreatment instead of 14 days. Similarly, there may have been different outcomes for those who had previous exposure to telehealth prior to treatment initiation with buprenorphine. Therefore, we conducted analyses within (1) those who used telehealth in the 2 years before initiating buprenorphine treatment and (2) those who were telehealth-naïve within the VHA at the start of treatment.

\section{RESULTS}

\section{Characteristics of Sample}

There were 28,791 Veterans in the final study sample (Fig. 1), of which $92.9 \%$ were males, $81.2 \%$ were white, and $48.8 \%$ were between the ages of 25 and 44 years at the time of their initial buprenorphine prescription within the VHA (Table 1). Psychiatric co-morbidities most frequently identified included depression $(73.2 \%)$, anxiety disorder $(60.9 \%)$, and posttraumatic stress disorder (39.8\%).

\section{SUD and Mental Health Encounters}

An estimated $3.2 \%$ and $5.3 \%$ of Veterans had telehealth for a SUD and mental health encounter, respectively, documented at least once during the course of their buprenorphine treatment. The median time from buprenorphine initiation to first telehealth was 13 days (IQR: 1-54 days) for SUD and 18 days (IQR: 3-65 days) for mental health encounters. The distribution and frequencies of primary stop code types are presented in Appendix C. The use of telehealth for both SUD and mental health encounters increased over time (Appendix D).

\section{Treatment Discontinuation}

The overall proportion of patients who discontinued buprenorphine within 1 year of starting was $66.5 \%(n=19,143)$, and the median time to discontinuation within this group was 51 days (IQR: 16-134 days). Older age, black race, and homelessness were more associated with treatment discontinuation, while discontinuation was less likely in rural Veterans (Table 1). The risk of discontinuation among those with a documented telehealth encounter was 0.69 (95\% CI: 0.60, 0.78 ) times that of someone with only an in-person SUD encounter (Table 2). The results of telehealth for mental health encounters were similar to those observed for SUD (aHR: $0.69 ; 95 \% \mathrm{CI}: 0.62,0.76)$. In sensitivity analyses, there was no considerable variation in associations between encounter types for SUD encounters or mental health encounters with treatment retention (Appendix E). When limiting the analysis to the 10 sites that most frequently used telehealth, the associations between telehealth use and treatment retention still remained. 
Initial Cohort

$\mathrm{N}=33,088$

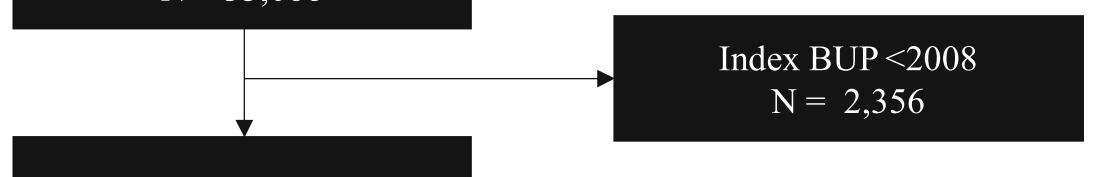

Index BUP $\geq 2008$

$\mathrm{N}=30,732$

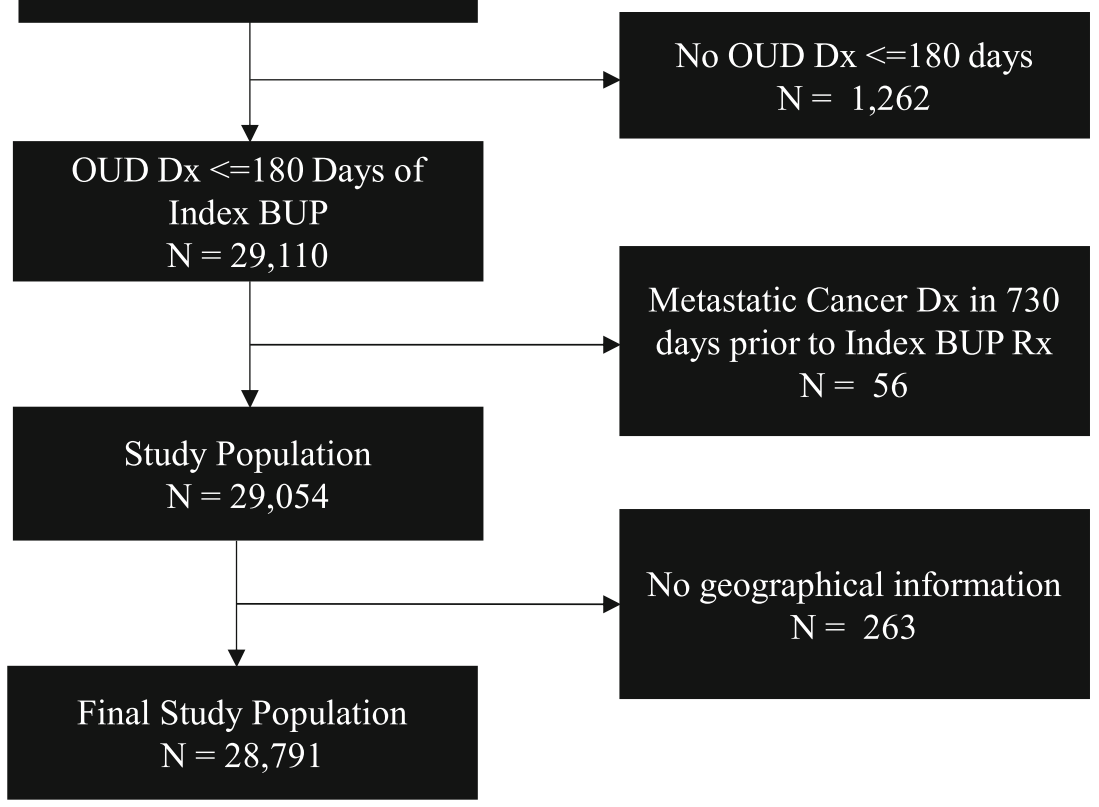

Figure 1 Flow chart of the study sample, Veterans treated for OUD with buprenorphine in the VHA, 2008-2017.

\section{Evaluation of Effect Modification}

There was no evidence that rurality was an effect modifier; we found no significant difference between the effects of inperson and telehealth encounters for SUD $(p=0.158)$ or for mental health $(p=0.126)$ by rural-urban designation (Fig. 2A). There was evidence of effect modification by telehealth and in-person visit categorization for both SUD $(p<0.001)$ and mental health $(p<0.001)$ encounters. The risk of treatment discontinuation appeared to be lower among those with telehealth only compared to in-person only for both SUD (aHR: $0.48,95 \%$ CI: $0.37,0.62$ ) and mental health encounters (aHR: 0.46; 95\%CI: 0.33, 0.65) (Fig. 2B).

\section{DISCUSSION}

Prior to the COVID-19 pandemic, telehealth was gaining momentum as a mechanism for providing mental health care while overcoming numerous logistical barriers. We studied whether telehealth, when employed during OUD management with buprenorphine, could be comparable to in-person counseling and services in the pre-pandemic setting. The VHA may serve as an optimal source for assessing the delivery of care through telehealth as it initiated this technology over 20 years ago, while augmenting its use since 2004 particularly for rural Veterans. ${ }^{24,34}$ Though our study found relatively low use overall, the VHA still offers numerous individual and group programs designed for mental health and substance disorder management. ${ }^{35}$ In this study, we examined a cohort of nearly 30,000 Veterans to identify telehealth encounters during OUD management within the VHA system and to evaluate potential heterogeneity in effects by urban-rural designation.

One of the key findings in this study is that for both SUD and mental health encounters, engagement with telehealth was associated with a lower risk of treatment discontinuation. Telehealth has been touted as a means to rapidly overcome several barriers and challenges in mental health care. ${ }^{21-23}$ The American Psychiatric Association identified numerous advantages telehealth offers including improving access to mental health specialty care that may not be otherwise available, helping integrate behavioral health care and primary care, reducing delays in patient care while improving continuity of care and follow up, and reducing transportation barriers. ${ }^{36} \mathrm{As}$ buprenorphine is an effective means to reducing overdoses and adverse events, ${ }^{37}$ perhaps providing additional services such as programs, therapies, counseling, or medication management through telehealth as a medium for those who need it the most is a viable option for facilities and providers to consider to improve treatment retention. 
Table 1 Baseline Characteristics of Veterans Treated for OUD with Buprenorphine in the VHA with Risk of Treatment Discontinuation up to 1 Year from Treatment Initiation, 2008-2017

\begin{tabular}{|c|c|c|c|}
\hline Demographic and clinical characteristics & Total $(\%)(N=28,791)$ & uHR (95\% CI) & $p$ value \\
\hline \multicolumn{4}{|l|}{ Gender } \\
\hline Female & $2044(7.1)$ & Ref & \\
\hline Male & $26,747(92.9)$ & $0.97(0.91-1.02)$ & 0.22 \\
\hline \multicolumn{4}{|l|}{ Age (years) } \\
\hline $18-24$ & $1121(3.9)$ & Ref & \\
\hline $25-44$ & $14,044(48.8)$ & $1.52(1.38,1.66)$ & $<0.001$ \\
\hline $45-64$ & 12,059 (41.9) & $1.15(1.07,1.23)$ & $<0.001$ \\
\hline$\geq 65$ & $1567(5.4)$ & $1.09(1.02,1.16)$ & 0.02 \\
\hline \multicolumn{4}{|l|}{ Race } \\
\hline White & $23,389(81.2)$ & Ref & \\
\hline Black & $3587(12.5)$ & $1.30(1.30,1.42)$ & $<0.001$ \\
\hline Other & $667(2.3)$ & $1.04(0.95,1.15)$ & 0.39 \\
\hline Unknown/missing & $1148(4.0)$ & $1.03(0.95,1.11)$ & 0.48 \\
\hline \multicolumn{4}{|l|}{ Ethnicity } \\
\hline Hispanic & $1455(5.1)$ & Ref & \\
\hline Non-Hispanic & $26,557(92.2)$ & $0.93(0.97,0.99)$ & 0.02 \\
\hline Unknown/missing & $779(2.7)$ & $0.94(0.84,1.04)$ & 0.23 \\
\hline \multicolumn{4}{|l|}{ Indication for homelessness/unstable housing } \\
\hline No & $20,468(71.1)$ & Ref & \\
\hline Yes & $8323(28.9)$ & $1.35(1.31,1.40)$ & $<0.001$ \\
\hline \multicolumn{4}{|l|}{ Residential designation } \\
\hline Urban & $25,516(88.6)$ & Ref & \\
\hline Rural & $3275(11.4)$ & $0.84(0.80,0.88)$ & $<0.001$ \\
\hline \multicolumn{4}{|l|}{ Distance to closest primary care facility } \\
\hline $0-19$ miles & $22,431(77.9)$ & Ref & \\
\hline$\geq 20$ miles & $6360(22.1)$ & $0.88(0.85,0.92)$ & $<0.001$ \\
\hline \multicolumn{4}{|l|}{ Distance to closest secondary care facility } \\
\hline $0-19$ miles & $12,456(43.3)$ & Ref & \\
\hline$\geq 20$ miles & $16,303(56.6)$ & $0.90(0.87,0.92)$ & $<0.001$ \\
\hline Ünknown/missing & $32(0.1)$ & $1.00(0.66,1.50)$ & 0.99 \\
\hline \multicolumn{4}{|l|}{ Drive time to closest primary care facility } \\
\hline $0-29 \mathrm{~min}$ & $23,746(82.5)$ & Ref & \\
\hline$\geq 30 \mathrm{~min}$ & $5045(17.5)$ & $0.88(0.85,0.91)$ & $<0.001$ \\
\hline \multicolumn{4}{|l|}{ Drive time to closest secondary care facility } \\
\hline $0-29 \min$ & $13,952(48.5)$ & Ref & \\
\hline$\geq 30 \mathrm{~min}$ & $14,807(51.4)$ & $0.90(0.88,0.93)$ & $<0.001$ \\
\hline Ūnknown/missing & $32(0.1)$ & $1.01(0.67,1.51)$ & 0.98 \\
\hline Treatment initiation era & & & \\
\hline 2008-2009 & 3904 (13.6) & Ref & \\
\hline 2010-2011 & $5303(18.4)$ & $0.95(0.91,1.00)$ & 0.06 \\
\hline $2012-2013$ & $5915(20.5)$ & $0.96(0.91,1.01)$ & 0.08 \\
\hline 2014-2015 & $6383(22.2)$ & $0.94(0.90,0.99)$ & 0.02 \\
\hline 2016-2017 & $7286(25.3)$ & $1.01(0.96,1.06)$ & 0.70 \\
\hline Clinical characteristics $(\mathrm{Ref}=\mathrm{No})$ & & & \\
\hline Co-morbidities & & & \\
\hline AIDS/HIV & $268(0.9)$ & $1.29(1.12,1.49)$ & $<0.001$ \\
\hline Cardiac arrhythmia & $2087(7.2)$ & $1.11(1.05,1.17)$ & $<0.001$ \\
\hline Chronic obstructive pulmonary disease & 3917 (13.6) & $1.02(0.98,1.06)$ & 0.33 \\
\hline Congestive heart failure & $639(2.2)$ & $1.07(0.97,1.18)$ & 0.17 \\
\hline Diabetes (with complications) & $925(3.2)$ & $1.02(0.94,1.11)$ & 0.58 \\
\hline Diabetes (without complications) & $2581(9.0)$ & $1.01(0.96,1.06)$ & 0.70 \\
\hline Hypertension (with complications) & $475(1.6)$ & $1.19(1.07,1.33)$ & 0.002 \\
\hline Hypertension (without complications) & $9325(32.4)$ & $0.96(0.93,0.99)$ & 0.011 \\
\hline Liver disease & $4392(15.3)$ & $1.15(1.10,1.19)$ & $<0.001$ \\
\hline Malignancy & $792(2.8)$ & $0.97(0.88,1.05)$ & 0.428 \\
\hline Myocardial infarction & $305(1.1)$ & $1.08(0.94,1.24)$ & 0.256 \\
\hline Non-metastatic cancer & $695(2.4)$ & $0.95(0.87,1.05)$ & 0.321 \\
\hline Obesity & 3300 (11.5) & $0.95(0.91,0.99)$ & 0.022 \\
\hline Peripheral vascular disease & $701(2.4)$ & $1.00(0.91,1.10)$ & 0.955 \\
\hline Renal disease & $559(1.9)$ & $1.12(1.01,1.24)$ & 0.026 \\
\hline Weight loss & $1034(3.6)$ & $1.13(1.05,1.21)$ & 0.002 \\
\hline Psychiatric co-morbidities & & & \\
\hline Attention-deficit/hyperactivity disorder & $1653(5.7)$ & $1.09(1.03,1.16)$ & 0.004 \\
\hline Adjustment & $4410(15.3)$ & $1.14(1.10,1.19)$ & $<0.001$ \\
\hline Anxiety disorder & $17,529(60.9)$ & $1.13(1.10,1.17)$ & $<0.001$ \\
\hline Bipolar disorder & $5317(18.5)$ & $1.16(1.12,1.21)$ & $<0.001$ \\
\hline Depression & $21,084(73.2)$ & $1.18(1.14,1.22)$ & $<0.001$ \\
\hline Personality disorder & $2283(7.9)$ & $1.24(1.18,1.30)$ & $<0.001$ \\
\hline Post-traumatic stress disorder & $11,459(39.8)$ & $1.21(1.18,1.25)$ & $<0.001$ \\
\hline Schizophrenia & $1701(5.9)$ & $1.27(1.20,1.35)$ & $<0.001$ \\
\hline Other substance use dependence & & & \\
\hline Alcohol & $10,475(36.4)$ & $1.23(1.19,1.26)$ & $<0.001$ \\
\hline Stimulants & $6103(21.2)$ & $1.33(1.28,1.37)$ & $<0.001$ \\
\hline
\end{tabular}


Table 1. (continued)

\begin{tabular}{|c|c|c|c|}
\hline Demographic and clinical characteristics & Total $(\%)(N=28,791)$ & UHR (95\% CI) & $p$ value \\
\hline Cannabis & $3685(12.8)$ & $1.30(1.25,1.36)$ & $<0.001$ \\
\hline Cocaine & $5436(18.9)$ & $1.35(1.31,1.40)$ & $<0.001$ \\
\hline Hallucinogen & $123(0.4)$ & $1.46(1.19,1.79)$ & $<0.001$ \\
\hline Nicotine & $14,852(51.6)$ & $1.08(1.05,1.11)$ & $<0.001$ \\
\hline Sedative & $2589(9.0)$ & $1.26(1.17,1.29)$ & $<0.001$ \\
\hline \multicolumn{4}{|l|}{ Recent medication use } \\
\hline Antidepressants & $18,682(64.9)$ & $1.08(1.05,1.11)$ & $<0.001$ \\
\hline Antipsychotics & $6544(22.7)$ & $1.18(1.15,1.22)$ & $<0.001$ \\
\hline Anxiolytics & $9683(33.6)$ & $1.05(1.02,1.08)$ & 0.001 \\
\hline Mood stabilizers & $10,065(35.0)$ & $1.12(1.09,1.16)$ & $<0.001$ \\
\hline Opioids & $10,941(38.0)$ & $1.01(0.98,1.04)$ & 0.72 \\
\hline Stimulants & $1011(3.5)$ & $0.99(0.92,1.07)$ & 0.77 \\
\hline \multicolumn{4}{|l|}{ Healthcare utilization } \\
\hline \multicolumn{4}{|l|}{ Inpatient admission } \\
\hline Any reason & $13,176(45.8)$ & $1.44(1.40,1.48)$ & $<0.001$ \\
\hline Mental health & $12,436(43.2)$ & $1.46(1.42,1.51)$ & $<0.001$ \\
\hline Suicide or self-harm & $2886(10.0)$ & $1.33(1.27,1.39)$ & $<0.001$ \\
\hline Substance use & $11,455(39.8)$ & $1.46(1.42,1.50)$ & $<0.001$ \\
\hline Urgent care use & $4382(15.2)$ & $1.13(1.09,1.18)$ & $<0.001$ \\
\hline \multicolumn{4}{|l|}{ Emergency department use } \\
\hline Suicide or self-harm & $2242(7.8)$ & $1.33(1.27,1.40)$ & $<0.001$ \\
\hline Substance use & $8293(28.8)$ & $1.24(1.20,1.27)$ & $<0.001$ \\
\hline
\end{tabular}

From the current design and approach, we can speculate that there may be two additional reasons for the observed associations. First, there may be additional individual-, facility-, or system-level factors that not only drive the decision to incorporate or implement telehealth services, but may also be associated with treatment retention. As previous work has demonstrated, there is significant diversity in OUD management and care across the country such as buprenorphine implementation, geographical variation of OUD within nonVHA settings, and types of behavioral interventions in addition to buprenorphine management. ${ }^{10,38} \mathrm{We}$ attempted to account for a potential selection bias issue by examining the association within a cohort that was previously exposed to telehealth, and then separately in one that was telehealth-naïve at the start of buprenorphine treatment. We could find no significant difference between the two, and overall associations in the sensitivity analysis were similar to the primary analysis.

Second, though we were not examining a causal relationship between telehealth or in-person visits with treatment retention, we still proposed that these encounters may influence buprenorphine treatment as an outcome. The inherent challenge with this approach is that rather than telehealth encounters motivating treatment retention, medication adherence and longer treatment retention times may be motivating the incorporation of telehealth into SUD management (i.e., a reverse causal relationship). To address this issue, we analyzed Veterans who stayed in treatment for at least 28 days (thereby examining encounters after 4 weeks of continuous treatment) and found no difference in the exposure and outcome associations. This, however, does not preclude the possibility that Veterans who were doing better may have been preferentially selected for telehealth.
We also found no evidence of effect modification by ruralurban designation when comparing telehealth and in-person visits, only that the risk of discontinuation was lower among rural Veterans compared to urban Veterans who had telehealth. This may seem paradoxical to the literature or other documentation that consistently report rurality as a risk factor for adverse outcomes of OUD or as a barrier to buprenorphine treatment and other mental health care. ${ }^{11,20,39-43}$ Two phenomena may be taking place within both the VHA system and rural Veterans. First, we followed a cohort of Veterans who were diagnosed with OUD and initiated buprenorphine treatment. This may present some selection bias, particularly if the adoption of buprenorphine as a line of treatment for OUD occurs less frequently in rural areas; those who start buprenorphine in some areas may be systematically different or have different access to care from those who do not start buprenorphine. Second, in order to address urban-rural disparities for Veterans and improve health outcomes, the VHA expanded or increased services such as the flexibility to incorporate nonVHA providers into coordinated care or the incorporation of telehealth as examined in this study. ${ }^{44-49}$ We suspect that some of these efforts by the VHA could explain some of the improvements in access and health outcomes, particularly in rural areas.

In this study of pre-pandemic use of telehealth, we were able to assess the extent to which this modality was used for both mental health and SUD services. The use of telehealth increased each year since the start of the study period for both mental health and substance use disorder services. Despite the potential promise of telehealth, we found that it was relatively rarely used; only $3 \%$ and $5 \%$ of Veterans had telehealth for a SUD and mental health encounter, respectively. However, it is important to recognize the role that the VHA plays in mental 
Table 2 Association Between Encounter Type and Risk of Discontinuation within 1 Year from Buprenorphine Treatment Initiation Among Veterans Treated in the VHA, 2008-2017

\begin{tabular}{|c|c|c|c|c|c|}
\hline $\begin{array}{l}\text { Telehealth } \\
\text { categorization }\end{array}$ & $\begin{array}{l}\text { Person } \\
\text { days at } \\
\text { risk }\end{array}$ & $n$ & IR & $\begin{array}{l}\text { uHR } \\
\text { (95\% } \\
\text { CI) }\end{array}$ & $\begin{array}{l}\text { aHR } \\
(95 \% \\
\text { CI) })^{*}\end{array}$ \\
\hline \multicolumn{6}{|c|}{ Primary analysis: encounter type for substance use dependence } \\
\hline $\begin{array}{l}\text { In-person en- } \\
\text { counter only }\end{array}$ & $3,646,110$ & 13,716 & 3.76 & Ref & Ref \\
\hline $\begin{array}{l}\text { Telehealth } \\
\text { encounter }\end{array}$ & 108,755 & 236 & 2.17 & $\begin{array}{l}0.63 \\
(0.56 \\
0.72)\end{array}$ & $\begin{array}{l}0.69 \\
(0.60, \\
0.78)\end{array}$ \\
\hline \multicolumn{6}{|c|}{ Secondary analysis: encounter type for mental health } \\
\hline $\begin{array}{l}\text { In-person en- } \\
\text { counter only }\end{array}$ & $4,215,366$ & 16,554 & 3.93 & Ref & Ref \\
\hline $\begin{array}{l}\text { Telehealth } \\
\text { encounter }\end{array}$ & 153,894 & 357 & 2.32 & $\begin{array}{l}0.64 \\
(0.58 \\
0.71)\end{array}$ & $\begin{array}{l}0.69 \\
(0.62, \\
0.76)\end{array}$ \\
\hline
\end{tabular}

VHA, Veterans Health Administration; $n$, number of discontinuations; IR, incidence rate (discontinuation per 1000 person-days); uHR, unadjusted hazard ratio; aHR, adjusted hazard ratio

* Substance use visit model adjusted for demographics year of buprenorphine treatment initiation, age, gender, race, homelessness, rurality, drive distance to primary care facility), clinical co-morbidities (HIV/AIDS, arrhythmia, coronary heart failure, hypertension w/o complications, liver disease, obesity, adjustment disorders, anxiety, bipolar, PTSD), concurrent substance dependence (alcohol, stimulants, cannabis, cocaine, hallucinogens, nicotine, sedatives), medications (antidepressants, antipsychotics), and healthcare utilization (any inpatient admission, psychiatric inpatient admission, urgent care use, emergency department visit for overdose, emergency department visit for suicide/self-harm)

t Mental health visit model adjusted: Demographics (year of buprenorphine treatment initiation, age, gender, race, homelessness, rurality, drive distance to primary care facility), clinical co-morbidities (hypertension w/o complications, liver disease, obesity, adjustment disorders, anxiety, bipolar, depression, PTSD), concurrent substance dependence (alcohol, stimulants, cannabis, cocaine, hallucinogens, nicotine, sedatives), medications (antidepressants, mood stabilizers), and healthcare utilization (any inpatient admission, psychiatric inpatient admission, urgent care use, emergency department visit for overdose, emergency department visit for suicide/self-harm)

$\$$ Telehealth encounters include telehealth services alone or in combination with in-person services. Telehealth is defined as encounters that used video-specific delivery of care

health and SUD care for Veterans beyond the delivery of telehealth as a mode of care. There are factors that are

A. Rural-Urban Designation

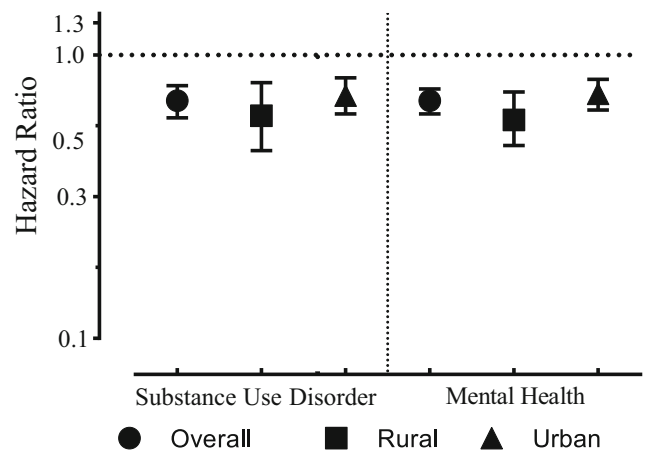

associated with qualifying someone to access VHA services. ${ }^{50}$ Substance disorders, mental health conditions, service-related injuries, and homelessness are positively associated, and there are programs and outreach efforts targeting Veterans who have such conditions. ${ }^{50-52}$ This may also lead to a selection of Veterans who are diagnosed and treated for OUD that might additionally utilize services such as therapy and counseling.

The COVID-19 pandemic has resulted in economic and psychosocial challenges, which further exacerbated the opioid crisis and mental health inequities. ${ }^{53}$ Due to the disruption in social services, there were numerous changes that took place to reduce barriers to receiving care after the onset of the pandemic. Some of these included expanding telehealth use for OUD management (by relaxing Ryan Haight Act restrictions), waiving the Health Insurance Portability and Accountability Act penalties for good faith use of telehealth, increasing access to telehealth for buprenorphine management and takehome medications, and covering additional telehealth services through Medicare ${ }^{54}$ In order to increase telehealth services for SUD, we will still need to investigate the patient-, provider-, and facility-level characteristics that lead to telehealth incorporation. We investigated additional elements of buprenorphine prescribing for our sample: provider specialization for prescribers and the use of telehealth for Veterans treated for OUD in primary care. Interestingly, $18 \%$ of providers who prescribed buprenorphine in this sample were primary care or general internal medicine providers, whereas the vast majority (74\%) of buprenorphine prescriptions were written by mental health providers. An estimated $27 \%$ of the general internal medicine and/or primary care providers also had a mental health sub-specialization and $11 \%$ had an addiction specialization. The second area that was further examined was the use of telehealth within the primary care setting for these patients. When we limited stop codes to only account for primary care or general medicine clinics, only $0.2 \%$ of patients had a telehealth encounter within this setting during follow-up. This presents an opportunity for expansion as increasing clinical

B. Telehealth and In-Person Combination

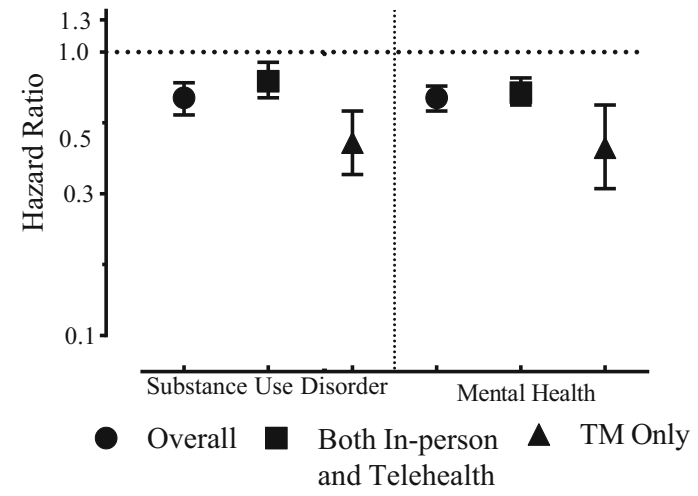

* Reference Group = In-person encounters only

Figure 2 Evaluation of telehealth by urban/rural designation and telehealth/in-person combination. 
capacity among general medicine and primary care providers would rapidly increase the availability of buprenorphine providers and could reduce inequities in access to care.

There are some limitations to this study. First, we relied on administrative data for exposure assessment, co-morbidities and other characteristics, and outcome ascertainment of buprenorphine treatment. This may have underestimated some treatment effects, despite our efforts to address concerns through multiple sensitivity analyses. There may also be significant variation in access and availability of treatments and services across facilities, potentially confounding some of the observed associations. There was no significant difference in our outcomes when we limited the analyses to facilities that more rigorously incorporated telehealth. Finally, the appropriate time to discontinuation is highly variable and debatable. We chose a timeline of 1 year, though the length of time on buprenorphine is a patient-centered concern and decision. As a result, we might have treated discontinuation as a "failure" or gap in healthcare when, in fact, discontinuation within 1 year may have been related to treatment success in some cases.

In conclusion, we found evidence that engagement with telehealth may improve treatment retention with buprenorphine for Veterans treated for OUD. Future research may elucidate the types of telehealth services provided for those with OUD, and whether telehealth for buprenorphine prescribing may be effective in improving retention. Treatment retention for OUD continues to be a challenge, and providers may consider alternative methods to keep their patients engaged during the course of treatment through services such as telehealth.

Corresponding Author: J. Priyanka Vakkalanka, PhD, ScM; Department of Emergency Medicine, University of Iowa Carver College of Medicine, Iowa City, IA, USA (e-mail: priyanka-vakkalanka@uiowa. edu).

Supplementary Information The online version contains supplementary material available at https://doi.org/10.1007/s11606-02106969-1.

Funding This work was supported by the National Institute on Drug Abuse's R36 Drug Abuse Dissertation Grant (1R36DA050878-01), and a training grant and pilot grant provided by the Centers for Disease Control and Prevention/National Institute of Occupational Health and Safety/ Heartland Center for Occupational Health and Safety Education and Research Center (T42OH008491). Additional support was provided by the Health Services Research and Development Service, Department of Veterans Affairs. This material is the result of work supported with resources and the use of facilities at the Iowa City VA Health Care System.

Data Availability The United States Department of Veterans Affairs (VA) places legal restrictions on access to veteran's health care data, which includes both identifying data and sensitive patient information. The analytic data sets used for this study are not permitted to leave the VA firewall without a Data Use Agreement. This limitation is consistent with other studies based on VA data. However, VA data are made freely available to researchers behind the VA firewall with an approved VA study protocol.

\section{Declarations:}

Conflict of Interest: The authors declare that they do not have a conflict of interest.

Disclaimers: The views expressed in this article are those of the authors and do not necessarily reflect the position or policy of the Department of Veterans Affairs or the United States Government.

\section{REFERENCES}

1. Centers for Disease Control and Prevention. Understanding the Epidemic. Available at: https://www.cdc.gov/drugoverdose/epidemic/index.html\#resources. Accessed September 7, 2020.

2. Wilder CM, Miller SC, Tiffany E, Winhusen T, Winstanley EL, and Stein MD. Risk factors for opioid overdose and awareness of overdose risk among veterans prescribed chronic opioids for addiction or pain. J Addict Dis. 2016;35:42-51.

3. Teeters JB, Lancaster CL, Brown DG, and Back SE. Substance use disorders in military veterans: prevalence and treatment challenges. Substance abuse and rehabilitation. 2017;8:69-77.

4. Bohnert AS, Ilgen MA, Galea S, McCarthy JF, and Blow FC. Accidental poisoning mortality among patients in the Department of Veterans Affairs Health System. Med Care. 2011;49:393-6.

5. McCarthy JF, Valenstein M, Kim HM, Ilgen M, Zivin K, and Blow FC. Suicide mortality among patients receiving care in the veterans health administration health system. Am J Epidemiol. 2009;169:1033-8.

6. National Institute on Drug Abuse. Substance Use and Military Life DrugFacts. Available at: https://www.drugabuse.gov/publications/drugfacts/substance-use-military-life\#ref. Accessed October 5, 2020.

7. Tsai $\mathrm{J}$ and Rosenheck RA. Risk factors for homelessness among US veterans. Epidemiol Rev. 2015;37:177-95.

8. Weber J, Lee RC, and Martsolf D. Understanding the health of veterans who are homeless: A review of the literature. Public Health Nurs. 2017;34:505-511.

9. Iheanacho T, Stefanovics E, and Rosenheck R. Opioid use disorder and homelessness in the Veterans Health Administration: The challenge of multimorbidity. J Opioid Manag. 2018;14:171-182.

10. Gordon AJ, Trafton JA, Saxon AJ, Gifford AL, Goodman F, Calabrese VS, et al. Implementation of buprenorphine in the Veterans Health Administration: results of the first 3 years. Drug Alcohol Depend. 2007;90:292-6.

11. Wyse JJ, Gordon AJ, Dobscha SK, Morasco BJ, Tiffany E, Drexler K, et al. Medications for opioid use disorder in the Department of Veterans Affairs (VA) health care system: Historical perspective, lessons learned, and next steps. Subst Abus. 2018;39:139-144.

12. Substance Abuse and Mental Health Services Administration. Medication-Assisted Treatment (MAT). Available at: https://www. samhsa.gov/medication-assisted-treatment. Accessed October 5, 2020.

13. Rosenblum A, Cleland CM, Fong C, Kayman DJ, Tempalski B, and Parrino M. Distance traveled and cross-state commuting to opioid treatment programs in the United States. J Environ Public Health. 2011;2011:948789.

14. Haffajee RL, Bohnert ASB, and Lagisetty PA. Policy Pathways to Address Provider Workforce Barriers to Buprenorphine Treatment. Am J Prev Med. 2018;54:S230-s242.

15. Knudsen HK, Abraham AJ, and Oser CB. Barriers to the implementation of medication-assisted treatment for substance use disorders: the importance of funding policies and medical infrastructure. Eval Program Plann. 2011;34:375-81.

16. National Council for Behavioral Health. Factors that Influence Access to Medication-Assisted Treatment. Available at: http://www.behavioralhealthworkforce.org/wp-content/uploads/2019/10/Factors-that-Influence-MAT_Full-Report.pdf. Accessed October 5, 2020.

17. Simpson DD. A conceptual framework for drug treatment process and outcomes. J Subst Abuse Treat. 2004;27:99-121.

18. Center for Substance Abuse Treatment, Substance Abuse and Mental Health Services Administration, and U.S. Department of Health and Human Services. Medication-Assisted Treatment For Opioid Addiction in Opioid Treatment Programs. Available at: https://www.asam.org/docs/ advocacy/samhsa_tip43_matforopioidaddiction.pdf?sfvrsn=0. Accessed October 5, 2020. 
19. Teich J, Ali MM, Lynch S, and Mutter R. Utilization of Mental Health Services by Veterans Living in Rural Areas. J Rural Health. 2017;33:297304.

20. Sigmon SC. Access to Treatment for Opioid Dependence in Rural America: Challenges and Future Directions. JAMA Psychiatry. 2014;71:359-360.

21. Vanderpool D, An Overview of Practicing High Quality Telepsychiatry, in Mental Health Practice in a Digital World: A Clinicians Guide. Cham: Springer International Publishing; 2015:159-181.

22. Chan S, Parish M, and Yellowlees P. Telepsychiatry Today. Curr Psychiatry Rep. 2015;17:89.

23. Daschle T and Dorsey ER. The return of the house call. Ann Intern Med. 2015;162:587-8.

24. Adams SV, Mader MJ, Bollinger MJ, Wong ES, Hudson TJ, and Littman AJ. Utilization of Interactive Clinical Video Telemedicine by Rural and Urban Veterans in the Veterans Health Administration Health Care System. The Journal of Rural Health. 2019;35:308-318.

25. von Elm E, Altman DG, Egger M, Pocock SJ, Gøtzsche PC, and Vandenbroucke JP. The Strengthening the Reporting of Observational Studies in Epidemiology (STROBE) statement: guidelines for reporting observational studies. Lancet. 2007;370:1453-7.

26. Department of Veterans Affairs. Handbook for Coding Guidelines Available at: http://opf-labs.org/format-corpus/govdocs 1-error-pdfs/error_set_1/316267.pdf. Accessed February 28, 2019.

27. National Association of Veteran Affairs Optometrists. FY14 Active DSS Identifiers. Available at: https://www.navao.org/wp-content/uploads/ 2016/02/FY14-Active-DSS-Identifiers.pdf. Accessed February 9, 2021.

28. Peterson R, Gundlapalli AV, Metraux S, Carter ME, Palmer M, Redd A, et al. Identifying homelessness among veterans using VA administrative data: opportunities to expand detection criteria. PloS one. 2015; 10:e0132664

29. Manhapra A, Petrakis I, and Rosenheck R. Three-year retention in buprenorphine treatment for opioid use disorder nationally in the Veterans Health Administration. Am J Addict. 2017;26:572-580.

30. Economic Research Service and United States Department of Agriculture 2010 Rural-Urban Commuting Area (RUCA) Codes. Available at: https:// www.ers.usda.gov/data-products/rural-urban-commuting-area-codes/ documentation/. Accessed September 11, 2020.

31. Healthcare Cost and Utilization Project (HCUP). Clinical Classifications Software (CCS) for ICD-9-CM. Available at: https://www.hcup-us.ahrq.gov/toolssoftware/ccs/ccs.jsp. Accessed February 28, 2019.

32. Quan H, Sundararajan V, Halfon P, Fong A, Burnand B, Luthi JC, et al Coding algorithms for defining comorbidities in ICD-9-CM and ICD-10 administrative data. Med Care. 2005;43:1130-9.

33. Harris AH, Reeder RN, Ellerbe L, and Bowe T. Are VHA administrative location codes valid indicators of specialty substance use disorder treatment? J Rehabil Res Dev. 2010;47:699-708.

34. Darkins A. The growth of telehealth services in the Veterans Health Administration between 1994 and 2014: a study in the diffusion of innovation. Telemed J E Health. 2014;20:761-8.

35. U.S. Department of Veterans Affairs. Health Benefits - Mental Health Care. Available at: https://www.va.gov/healthbenefits/access/mental_health care.asp. Accessed February 11, 2021.

36. American Psychiatric Association. What is Telepsychiatry? Available at: https://www.psychiatry.org/patients-families/what-is-telepsychiatry. Accessed March 17, 2017.

37. Wakeman SE, Larochelle MR, Ameli O, Chaisson CE, McPheeters JT, Crown WH, et al. Comparative Effectiveness of Different Treatment Pathways for Opioid Use Disorder. JAMA Network Open. 2020;3:e1920622.

38. Timko C, Schultz NR, Cucciare MA, Vittorio L, and Garrison-Diehn C. Retention in medication-assisted treatment for opiate dependence: A systematic review. J Addict Dis. 2016;35:22-35.
39. Webster LR, Cochella S, Dasgupta N, Fakata KL, Fine PG, Fishman SM, et al. An Analysis of the Root Causes for Opioid-Related Overdose Deaths in the United States. Pain Medicine. 2011;12:S26-S35.

40. Centers for Disease Control and Prevention (CDC). Unintentional poisoning deaths-United States, 1999-2004. MMWR Morb Mortal Wkly Rep. 2007;56:93-6.

41. Lister JJ, Weaver A, Ellis JD, Himle JA, and Ledgerwood DM. A systematic review of rural-specific barriers to medication treatment for opioid use disorder in the United States. The American Journal of Drug and Alcohol Abuse. 2020;46:273-288.

42. Mackey K, Veazie S, Anderson J, Bourne D, and Peterson K, VA Evidencebased Synthesis Program Reports, in Evidence Brief: Barriers and Facilitators to Use of Medications for Opioid Use Disorder. Washington: Department of Veterans Affairs (US); 2019:

43. Johnson Q, Mund B, and Joudrey PJ. Improving Rural Access to Opioid Treatment Programs. The Journal of Law, Medicine \& Ethics. 2018;46:437-439.

44. Health Resources and Services Administration. Flex Rural Veterans Health Access Program. Available at: https://www.hrsa.gov/grants/ find-funding/hrsa-16-015. Accessed September 10, 2020.

45. Jacobs JC, Blonigen DM, Kimerling R, Slightam C, Gregory AJ, Gurmessa T, et al. Increasing Mental Health Care Access, Continuity, and Efficiency for Veterans Through Telehealth With Video Tablets. Psychiatr Serv. 2019;70:976-982.

46. Pearson K, Burgess A, Gale J, Coburn A, and Hansen A, Health Information Exchange: A Strategy for Improving Access for Rural Veterans in the Maine Flex Rural Veterans Health Access. 2016, University of Southern Maine, Muskie School of Public Service, Maine Rural Health Research Center: Portland, ME.

47. Weintraub E, Greenblatt $\mathrm{AD}$, Chang $\mathrm{J}$, Himelhoch $\mathrm{S}$, and Welsh $\mathrm{C}$. Expanding access to buprenorphine treatment in rural areas with the use of telemedicine. Am J Addict. 2018;27:612-617.

48. US Department of Veterans Affairs. Making Medication Assisted Treatment (MAT) Available at CBOCs Using Telemedicine. Available at: https:// www.queri.research.va.gov/visn initiatives/telemedicine.cfm. Accessed May 21, 2019

49. University of Pennsylvania Wharton School of Business. The VA Battles America's Veteran Mental Health Crisis with Telehealth Available at: https://publicpolicy.wharton.upenn.edu/live/news/2857-the-va-battles-americas-veteran-mental-health/for-students/blog/news. Accessed May 21, 2019.

50. United States Department of Veterans Affairs. Chapter 1 Health Care Benefits. Available at: https://www.va.gov/opa/publications/benefits_book/Chapter_1_Health_Care_Benefits.asp. Accessed January 18, 2021.

51. U.S. Department of Veterans Affairs. Veterans Experiencing Homelessness. Available at: https://www.va.gov/homeless/. Accessed February $13,2021$.

52. U.S. Department of Veterans Affairs. Mental Health. Available at: https:// www.mentalhealth.va.gov/. Accessed February 13, 2021.

53. Khatri U, DC Krawczyk, Lynch, Berk J, Samuels EA. These key telehealth policy changes would improve buprenorphine access while advancing health equity. Health Affairs blog. 2020. https://doi.org/10.1377/ hblog20200910.498716.

54. Lin LA, Fernandez AC, and Bonar EE. Telehealth for Substance-Using Populations in the Age of Coronavirus Disease 2019: Recommendations to Enhance Adoption. JAMA Psychiatry. 2020;77:1209-1210.

Publisher's Note: Springer Nature remains neutral with regard to jurisdictional claims in published maps and institutional affiliations. 